\title{
Responses of Macaque V1 Neurons to Binocular Orientation Differences
}

\author{
Holly Bridge ${ }^{1}$ and Bruce G. Cumming ${ }^{2}$ \\ 1 University Laboratory of Physiology, Oxford, OX1 3PT, United Kingdom, and 2Laboratory of Sensorimotor Research, \\ National Eye Institute, National Institutes of Health, Bethesda, Maryland 20892-4435
}

\begin{abstract}
Interocular differences in orientation occur during binocular viewing of a surface slanted in depth. These orientation disparities could be exploited by the visual system to provide information about surface slant, but gradients of positional disparity provide an equally effective means to the same end. We examined the encoding of orientation disparities in V1 neurons that were recorded from two awake fixating monkeys. Monocular orientation selectivity was measured separately in each eye. Although the preferred monocular orientation in the left and right eyes was highly correlated ( $r=0.98), 19$ of 61 cells showed a significant interocular difference in preferred orientation (IDPO). By itself, an IDPO does not imply a specific binocular selectivity for orientation differences. We therefore examined the response to 25 binocular combinations of orientations by pairing each of five orientations in one eye with five in the other. Forty-four of 64 neurons showed responses that re-
\end{abstract}

flected the monocular orientation tuning selectivity; the preferred orientation disparity changed when the monocular orientation was changed in either eye. The remaining third (20 of 64) responded to a consistent orientation disparity in a way that was not simply predictable from monocular orientation selectivity. However, nearly all of these neurons were selective for positional disparity, and several characteristics of the responses suggest that the apparent selectivity for orientation disparities was just a consequence of the positional disparity sensitivity. Neither the data presented here nor previous data from the cat (Blakemore et al., 1972; Nelson et al., 1977) support the idea that a population of neurons early in the visual system has a separate encoding scheme for orientation disparities.

Key words: orientation disparity; positional disparity; energy model; cortical area V1; awake macaque; electrophysiology
When a frontoparallel plane is viewed with both eyes, lines on the plane project to images with the same orientation in both eyes. However, when the plane is tilted, the orientations of the elements are no longer matched in the two eyes; there is an interocular orientation difference (orientation disparity). Wheatstone (1838) found that, when observers were presented with lines of different orientation in the two eyes, the three-dimensional percept was a line slanted in depth. (Slant is used here to mean rotation out of the frontoparallel plane about a horizontal axis.) This observation does not provide any insight into the underlying mechanism for perceiving slant that might exploit the orientation disparity or the gradient of positional disparity along the extent of the lines in each eye.

Several psychophysical studies have attempted to determine whether human observers use these orientation disparities rather than positional disparities to detect surface slant (von der Heydt, 1978; Ninio, 1985; Mitchison and McKee, 1990; Cagenello and Rogers, 1993). Three methods have been used to try to distinguish between positional and orientation disparity mechanisms: (1) putting the two types of disparity into conflict (Ninio, 1985), (2) exploiting the fact that line elements of different orientations contain different amounts of orientation disparity (Mitchison and McKee, 1990; Cagenello and Rogers, 1993), and (3) attempting to introduce orientation disparities in the absence of consistent

\footnotetext{
Received Feb. 28, 2001; revised June 15, 2001; accepted June 20, 2001.

This work was supported by the Wellcome Trust. H.B. was a Christopher Welch Scholar. B.G.C. was a Royal Society University Research Fellow. We thank Andrew Parker for comments on this manuscript.

Correspondence should be addressed to Dr. H. Bridge, University Laboratory of Physiology, Parks Road, Oxford, OX1 3PT, UK. E-mail: holly.bridge@physiol.ox.ac.uk. Copyright (C) 2001 Society for Neuroscience $0270-6474 / 01 / 217293-10 \$ 15.00 / 0$
}

positional disparities (von der Heydt et al., 1980). No study has been able to manipulate orientation and position disparities independently (the geometrical relationship between them makes this impossible). For this reason, these psychophysical studies have not led to any clear consensus concerning the role of orientation disparities in the perception of slant.

Approaching the problem from a neurophysiological perspective allows a direct investigation of the underlying neural mechanism. Two distinct populations of neurons early in the visual system, one selective for positional differences and one selective for orientation disparities, would suggest that there are two different mechanisms for detecting slant. Blakemore et al. (1972) demonstrated that some binocular neurons in area 17 of the cat possess different preferred orientations in the two eyes. We refer to this neuronal property as an interocular difference in preferred orientation (IDPO), to distinguish it from orientation disparities, which are a stimulus attribute. Blakemore et al. (1972) suggested that neurons with IDPOs were selective for orientation disparities, and hence formed a "second neural mechanism for depth perception." Although Hubel and Wiesel (1973) disputed the experimental observations, they have subsequently been replicated both in area 17 (Nelson et al., 1977) and area 21a (Wieniawa-Narkiewicz et al., 1992) of the cat.

All of the above studies were performed with anesthetized animals, in which it is possible that some of the results merely reflected torsional rotation of the eyes under anesthetic. We therefore investigated orientation tuning of binocular neurons in the awake, fixating monkey.

Even if IDPOs do occur, they are insufficient to encode orientation disparities. A binocular interaction that depends on the 
A

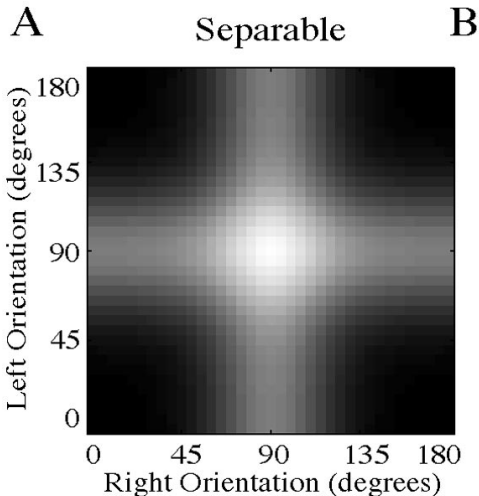

B

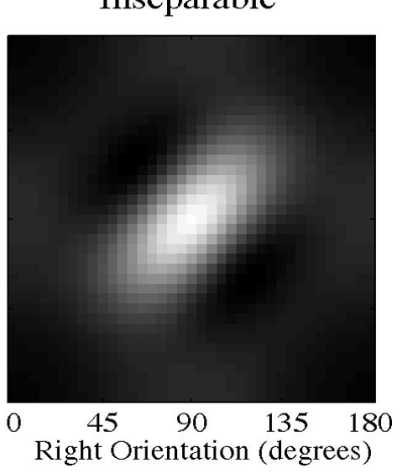

Figure 1. An illustration of the difference between separable and inseparable responses to orientation disparities. $A$ shows a response that is possible to predict from the two monocular orientation tuning curves (here the binocular response is simply the linear sum of the two monocular responses). In this case the preferred orientation disparity changes as the monocular orientations are changed. In contrast, the preferred orientation disparity in $B$ remains constant as the monocular orientations are changed. This is a response that cannot be separated into the left and right monocular tuning functions. Only such left-right inseparable responses yield tuning to orientation disparities that does not vary with monocular orientation.

orientation disparity is also required. Consider a binocular neuron that linearly sums activity from the input of the left and right eyes. Such a sum contains no information at all about the relationship between left and right images, so it is of no more value for depth perception than purely monocular signals. As Wieniawa-Narkiewicz et al. (1992) pointed out, all of the existing data on IDPOs are compatible with such an additive interaction; it is possible that binocular responses reflect the monocular orientation preferences without encoding any differences.

Figure 1 illustrates this distinction. It shows idealized responses to many combinations of stimulus orientation in the two eyes. The left panel is a simple summation of left and right responses, with no further binocular interaction. This produces a left-right separable response pattern (it can be separated into a function describing the responses of the left eye and the right eye. Adding these functions then describes the binocular responses.) The right panel shows an interaction that is specific to binocular orientation differences; the preferred orientation for one eye depends on the stimulus orientation in the other eye, and so it is left-right inseparable. Previous studies of binocular responses (Blakemore et al., 1972; Nelson et al., 1977; Wieniawa-Narkiewicz et al., 1992) have only manipulated stimulus orientation for one eye, keeping the orientation in the other eye constant. Such data are single cross sections through the surfaces shown in Figure 1 and so cannot distinguish the two response patterns.

Other properties of neuronal responses have been considered in an attempt to determine the usefulness of these signals for slant perception. Nelson et al. (1977) pointed out that the activity of most neurons was more strongly modulated by positional disparities than by orientation differences and that the bandwidth for tuning to orientation disparities was no narrower than that for monocular orientation. Although these observations suggest that IDPOs may be of limited value in encoding orientation disparities, they are compatible with interactions that are either leftright separable or left-right inseparable. Thus, the possible contribution of IDPOs to the physiological encoding of depth remains unclear. We therefore investigated the extent to which neurons in V1 of awake fixating monkeys encode orientation disparities. First, orientation tuning was examined in each eye monocularly. Then, binocular responses to a range of combinations of left and right orientations were measured to determine whether or not the binocular interaction between them was leftright separable.

\section{MATERIALS AND METHODS}

Animal training. Neurons were recorded from three hemispheres of two adult monkeys (Macaca mulatta), one male $(\mathrm{Hg})$ and one female $(\mathrm{Rb})$. All of the procedures that were performed on the animals complied with the United Kingdom Home Office regulations on animal experimentation. The animals were implanted with a head fixation post and scleral eye coils under general anesthetic (Judge et al., 1980; Cumming and Parker, 1999) and trained to fixate a binocular target for fluid reward.

Stimulus presentation. Stimuli were generated on a Silicon Graphics Indigo workstation and displayed on two cathode ray tube monitors. Both monitors were gamma corrected to produce a linear relationship between luminance and the computer gray level settings. The mean luminance was $42 \mathrm{~cd} . \mathrm{m}^{-2}$, contrast was $99 \%$, and the frame rate was $72 \mathrm{~Hz}$. The monitors were viewed by the animal through two small mirrors (diameter, $18 \mathrm{~mm}) \sim 2 \mathrm{~cm}$ away from the eyes in a Wheatstone stereoscope configuration. The viewing distance to the monitors was $85 \mathrm{~cm}$, and at this distance a pixel on the screen corresponded to $1.0 \mathrm{arc}$ min of visual angle. The red signal was used to present stimuli to the left monitor, and the blue signal to the right monitor, which ensured accurate synchronization of the binocular images. The stimuli on both monitors were rendered in black-white monochrome (there was no actual color difference between the images presented to the two eyes).

The initial step in plotting receptive fields for the neurons was to find the preferred orientation using a moving bar. Minimum response fields were plotted using a flashing, high-contrast bar at this preferred orientation. The stimuli were centered on, but larger than, the minimum response field. Thus, our stimuli will have covered the receptive field even if our measures of minimum response field underestimated this.

The main stimulus used in these experiments was a circular patch of sine-wave grating that was drifted across the receptive field. When measuring disparity tuning, we also used random dot stereograms (RDS). Both types of stimulus were presented against a mid-gray background. The RDS consisted of equal numbers of black and white dots. In all experiments, the stimulus was presented in the receptive field for 2 sec, and spikes were counted over the entire duration. See Cumming and Parker (1999) for more details.

Unit recording. Recording was performed using tungsten in glass microelectrodes (Merrill and Ainsworth, 1972). The electrode was advanced through the dura and left to rest for a few minutes. Neurons were then found by first withdrawing the electrode until gray matter was no longer heard and then advancing through the gray matter of primary visual cortex. We examined the relationship between receptive field location and the location of our electrode penetrations over a $10 \times 10$ $\mathrm{mm}^{2}$ area. There was a clear systematic map that agreed with the known topography of macaque primary visual cortex.

Signals from the electrode were amplified (Bak Electronics, Mount Airy, MD) and filtered $(200 \mathrm{~Hz}$ to $5 \mathrm{kHz}$ ) before being digitized (32 $\mathrm{kHz}$ ) and stored to disk. A Datawave Discovery System was used to store the spike waveforms and eye position signals, which were then reanalyzed off-line using software designed in our laboratory.

After plotting receptive fields, we measured orientation tuning with a binocular grating at zero disparity. A circular patch of grating extending from 0.5 to $1^{\circ}$ beyond the minimum response field was used. [Full field gratings were avoided to reduce any stimulus-driven torsional eye movements (Howard et al., 1994).] The preferred spatial frequency was measured at the preferred orientation, and this spatial frequency was used for measuring monocular orientation tuning curves. The same set of seven orientations was presented to each eye, centered about the binocular preferred orientation (in six cases, only five orientations were used). Stimuli presented to the left and right eyes were interleaved, and each stimulus was presented a minimum of four times.

Once the monocular orientation preference had been established, binocular interactions were measured. A two-dimensional tuning curve was constructed in which left and right orientations were altered systematically and independently. Five orientations for each eye were chosen to span the range of orientations to which the neuron responded. Each of 
the 25 combinations was presented at least four times, and all conditions were interleaved.

Finally, selectivity for positional disparity was tested with one (or both) of two different techniques. First, a random dot stereogram was used in which disparity was added by shifting the horizontal position of the dots in opposite directions in the two eyes. A minimum of five different disparities, centered about zero, was used, and more values were used if necessary to characterize the tuning curve more thoroughly. Disparity tuning was also measured with sine-wave gratings, because this was the stimulus used in all of the other experiments. The position of the circular grating patch in both eyes was maintained, whereas the interocular phase difference was manipulated. Neurons were classified as disparity-selective if they were significant at the $5 \%$ level on a one-way ANOVA of firing rate with respect to disparity. In addition to this test for significance, a disparity discrimination index (DDI) was calculated using the following equation from Prince et al. (2001):

$$
D D I=\frac{R_{\max }-R_{\min }}{\left(R_{\max }-R_{\min }\right)+2 R M S_{\text {error }}},
$$

where $R_{\max }$ is the largest response rate on the curve, $R_{\min }$ is the minimum response, and $R M S_{\text {error }}$ is square root of the residual variance across the whole tuning curve. The square root of the firing rate was used as the response measure in all of these tests (see next section).

Analysis. Regression analysis was used to fit equations to the data. However, spike count variance increases with the mean count, violating an important condition for regression analysis. Therefore, all fitting was performed on the square root of the firing rates, which largely removes this dependence (Cumming and Parker, 2000; Prince et al., 2001). (Because the interval over which spikes were counted was fixed, this has the same effect as taking the square root of the counts.) Only fits that accounted for at least $75 \%$ of the variance in the square root firing rate were used for subsequent analysis. Tuning curves for both orientation and disparity were considered to have "significant tuning" if the neuronal firing rate was consistently modulated by the changing stimulus parameter. Such modulation was quantified by performing a one-way ANOVA of firing rate with respect to the changing parameter.

Monocular orientation tuning curves were tested for significant tuning with two different tests: first, using a one-way ANOVA; and second, using a test to ensure that a significant component of the modulated data is smooth. The latter test exploited a sequential $F$ test (described below) to test that the Gaussian curve was a better fit to the data than a horizontal straight line through the mean spike rate (averaged across all trials). Neurons were only included if they were significant at the $5 \%$ level on both the ANOVA and the sequential $F$ test. The analysis of monocular orientation differences required that the tuning in both eyes meet this criterion. Binocular interactions were examined in neurons that met the criterion for at least one eye.

The monocular tuning data were fit using a nonlinear least square algorithm (numerical algorithms groups) in two different ways. First, two independent Gaussians were fit, one for the left eye and one for the right eye:

$$
\begin{gathered}
\text { Left }=A_{1} e^{\left(\theta-\omega_{1}\right)^{2} / 2 \sigma_{\theta l}^{2}}+B_{1}, \\
\text { Right }=A_{\mathrm{r}} e^{\left(\theta-\omega_{\mathrm{r}}\right)^{2} / 2 \sigma_{\theta \mathrm{r}}^{2}}+B_{\mathrm{r}},
\end{gathered}
$$

where $A_{1}$ and $A_{\mathrm{r}}$ are the amplitudes for the left and right eye, $B_{1}$ and $B_{\mathrm{r}}$ are the baseline firing rates, $\omega_{1}$ and $\omega_{\mathrm{r}}$ are the preferred orientations in the left and right eyes, and $\sigma_{l}^{2}$ and $\sigma_{r}^{2}$ are the variances for the left and right tuning functions.

Second, the two sets of data were fit such that they were constrained to have the same preferred orientation, $\omega$ (i.e., $\omega_{1}$ and $\omega_{\mathrm{r}}$ are both replaced with $\omega)$. The fitted curves in this condition had one less parameter overall. To test whether there was a significant difference in the preferred orientation in the two eyes, a sequential $F$ test was performed to see whether allowing the two preferred orientations to be independent led to a significantly better fit.

Gaussian curves have been shown to be an adequate description of orientation tuning curves in primary visual cortex of both the cat and the monkey (Henry et al., 1978; Parker and Hawken, 1988). The majority of orientation tuning curves in this study could be fit with a Gaussian function, although a minority showed deviations from this consistent with those previously described by DeValois et al. (1982).

Fitting orientation disparity curves measured binocularly. If the monocular orientation tuning curves are well described by Gaussians and the responses to binocular combinations are left-right separable, these binocular responses can be described by the sum and product of two Gaussians:

Response $=A_{\mathrm{l}} e^{-\left(\theta-\omega_{\mathrm{l}}\right)^{2} / 2 \sigma_{\mathrm{I}}^{2}}+A_{\mathrm{r}} e^{-\left(\theta-\omega_{\mathrm{r}}\right)^{2} / 2 \sigma_{\mathrm{r}}^{2}}+A_{\mathrm{lr}} e^{-\left(\theta-\omega_{\mathrm{l}}\right)^{2} / 2 \sigma_{\mathrm{I}}^{2}-\left(\theta-\omega_{\mathrm{r}}\right)^{2} / 2 \sigma_{\mathrm{r}}^{2}}+D$,

where $A_{\text {lr }}$ determines the amplitude of the multiplicative component and $D$ is the baseline activity.

A simple modification to this equation allows it to fit left-right inseparable responses, where this represents a consistent response to orientation disparities. This is done by adding a rotation term so that the fitted response surface can be elongated along a diagonal. In achieving this, Equation 4 becomes:

$$
\text { Response }=A_{u} e^{-u^{2} / 2 \sigma_{u}^{2}}+A_{v} e^{-v^{2} / 2 \sigma_{v}^{2}}+A_{u v} e^{-u^{2} / 2 \sigma_{u}^{2}-v^{2} / 2 \sigma_{v}^{2}}+D,
$$

where

$$
\begin{gathered}
u=\left(\theta_{\mathrm{x}}-\theta_{\mathrm{L}}\right) \cos (\phi)+\left(\theta_{\mathrm{y}}-\theta_{\mathrm{R}}\right) \sin (\phi), \\
v=-\left(\theta_{\mathrm{x}}-\theta_{\mathrm{L}}\right) \sin (\phi)+\left(\theta_{\mathrm{y}}-\theta_{\mathrm{R}}\right) \cos (\phi) .
\end{gathered}
$$

$\mathrm{A}_{\mathrm{u}}, A_{\mathrm{v}}, A_{\mathrm{uv}}$, and $D$ are constants, $\sigma_{u}^{2}$ and $\sigma_{v}^{2}$ are the variances in the $u$ and $v$ directions, and $\phi$ is the angle through which the axes have been rotated about the point $\left(\theta_{\mathrm{L}}, \theta_{\mathrm{R}}\right)$. Because this involves adding one parameter to Equation 4, a sequential $F$ test (Draper and Smith, 1998) can be used to give a statistical measure of the need for the rotation term.

Fitting binocular orientation disparity data with the energy equation. In some cases, we found that neurons responded to more than one orientation disparity, and Equation 5 produced a poor description of the data. The form of the responses looked very similar to those responses described previously in studies of the interaction between left and right stimulus positions (Ohzawa et al., 1997). This is not surprising if the underlying mechanism is like the energy model; the interaction depends only on the results of convolving the stimulus with the receptive field in each eye. If changes in orientation produced systematic changes in the value of this convolution in a way that is similar to the changes elicited by position changes, then one would expect the pattern of binocular interaction to be similar. For this reason, we chose to describe these responses with an equation of the same form as that used by Ohzawa et al. (1997):

$$
\begin{aligned}
\text { Response } & =A_{\mathrm{l}} e^{-2 \mathrm{k}\left(\theta-\omega_{1}\right)^{2}}+A_{\mathrm{r}} e^{-2 \mathrm{k}\left(\theta-\omega_{\mathrm{r}}\right)^{2}} \\
+ & 2 A_{\mathrm{lr}} e^{-\mathrm{k}\left(\left(\theta-\omega_{1}\right)^{2}+\left(\theta-\omega_{\mathrm{r}}\right)^{2}\right)} \cos \left(2 \pi f\left(\left(\theta-\omega_{\mathrm{l}}\right)-\left(\theta-\omega_{\mathrm{r}}\right)\right)-\phi\right)+D,
\end{aligned}
$$

where $A_{\mathrm{u}}, A_{\mathrm{v}}, A_{\mathrm{uv}}, k$, and $D$ are constants, $\omega_{1}$ and $\omega_{\mathrm{r}}$ are the preferred orientations in the left and right eyes, $f$ is the spatial frequency of the cosine term, and $\phi$ is the phase of the cosine term. This cosine term is the main difference between this surface and the one described in Equation 5 , and it allows for multiple peaks or troughs in the surface, rather than the single peak afforded by the Gaussian. Both types of fitting to binocular orientation differences were fit using a Levenberg-Marquardt algorithm run in Matlab (MathWorks Inc., Natick, MA).

Use of sequential $\mathrm{F}$ tests. Sequential $F$ tests are used to test whether adding one or more parameters to a linear regression significantly improves the fit of a curve to the data (Draper and Smith, 1998). The residuals from the two fits to be compared are used to calculate the $F$ ratio, which is of the following form:

$$
F\left(p_{1}-p_{2}, n-p_{1}\right)=\frac{\left(r_{2}-r_{1}\right) /\left(p_{1}-p_{2}\right)}{r_{1} /\left(n-p_{1}\right)},
$$

where $r_{1}$ is the residual from the fit with a greater number of parameters $\left(p_{1}\right), r_{2}$ is the residual from the fit with fewer parameters $\left(p_{2}\right)$, and $n$ is the total number of trials. For each situation in which these were used, a Monte Carlo simulation was performed to ensure that the frequency with which the null hypothesis was rejected was appropriate for the significance criterion.

\section{RESULTS}

A total of $141 \mathrm{~V} 1$ neurons were recorded in the two animals. One hundred seven of these were tested for orientation tuning in each eye. The remainder showed weak orientation selectivity to the binocular stimulus or were lost before the monocular data were 
A

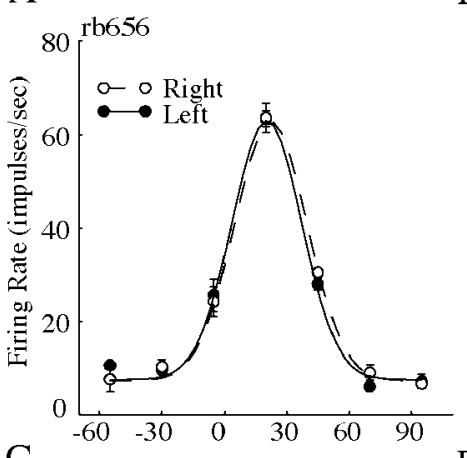

$\mathrm{C}$

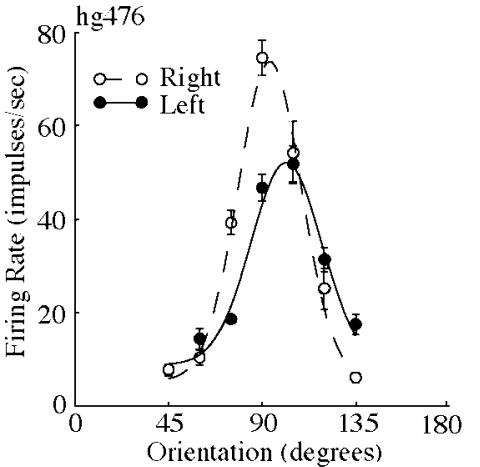

B

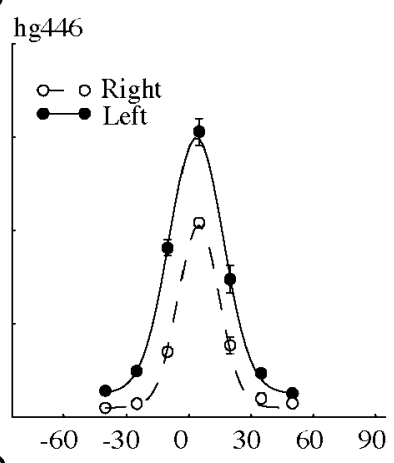

D

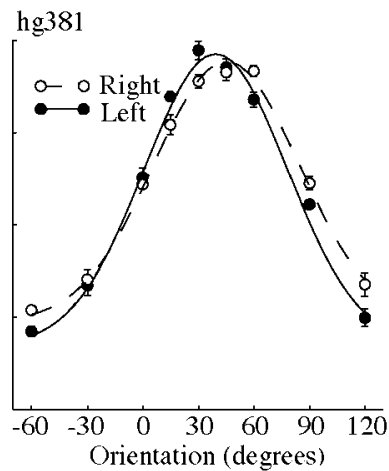

Figure 2. Sample monocular tuning curves for four cells. $A$ and $B$ show two cells in which the two eyes have almost identical tuning. In $A$, both eyes respond with almost exactly the same response rate, whereas in $B$ the maximum firing rate of the right eye is $\sim 75 \%$ of the maximum left eye firing rate. The left and right preferred orientations are significantly different in $C$ and $D$. The monocularly measured IDPOs are 7.9 and $5.6^{\circ}$, respectively.

collected. One hundred three of 107 neurons showed significant orientation selectivity in at least one eye. Seventy-seven of 103 showed significant tuning in both eyes and 26 of 103 were tuned only in one eye.

To compare the preferred orientation of each eye, it was necessary to restrict the analysis to neurons that were tuned in both eyes. Of these 77 neurons, 61 had tuning curves that were adequately described by Gaussian curves in both eyes. Of those neurons that could not be described by a Gaussian curve, the majority had multiple, smaller peaks away from the preferred orientation, as described previously by DeValois et al. (1982). Figure 2 shows monocular tuning curves for four neurons with the fitted Gaussian functions. The error bars show SEMs. $A$ and $B$ show IDPOs of 1.3 and $1.6^{\circ}$, respectively (neither significantly different from 0 ). $C$ and $D$ show significant IDPOs of 7.9 and $5.6^{\circ}$, respectively. Figure 3 summarizes the comparison of left and right preferred monocular orientations for all 61 neurons. Although the preferred orientation in the two eyes is highly correlated $(r=0.985)$, there is a scatter of IDPOs $(\mathrm{SD}=9.22)$. Nineteen of 61 of these cells have significantly different monocular orientations (sequential $F$ test; $p<0.05$ ).

Three neurons showed an unusual pattern of results in which the orientations that were excitatory in one eye were suppressive when presented to the other eye. An example is shown in Figure $4 A$, along with its disparity tuning curve $(B)$. Note that this shows suppression for near-zero disparities. This was true for the disparity tuning curves of all three neurons. This combination of response patterns suggests that the result of stimulation in one

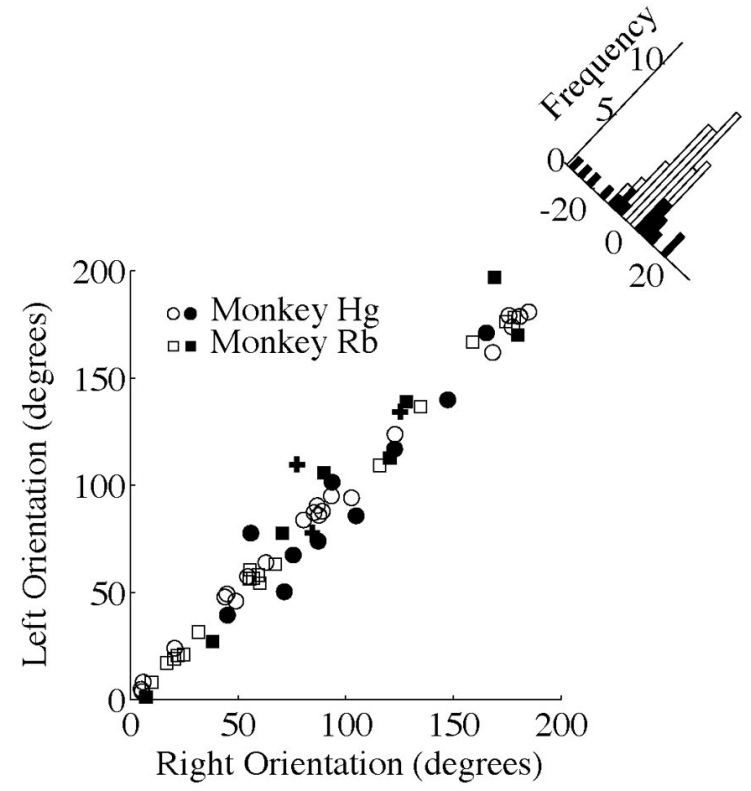

Figure 3. A scatterplot showing the very high correlation (0.985) between the left and right preferred orientations for the $61 \mathrm{~V} 1$ cells that are tuned to orientation in both eyes. Those cells showing a significant difference in their preferred orientation are shown with filled symbols, whereas those without any significant difference are shown with open symbols. The three cells in which one of the fitted Gaussian curves is inverted are shown with crosses. The range of differences in monocular preferred orientations (IDPOs) is summarized in the frequency histogram.
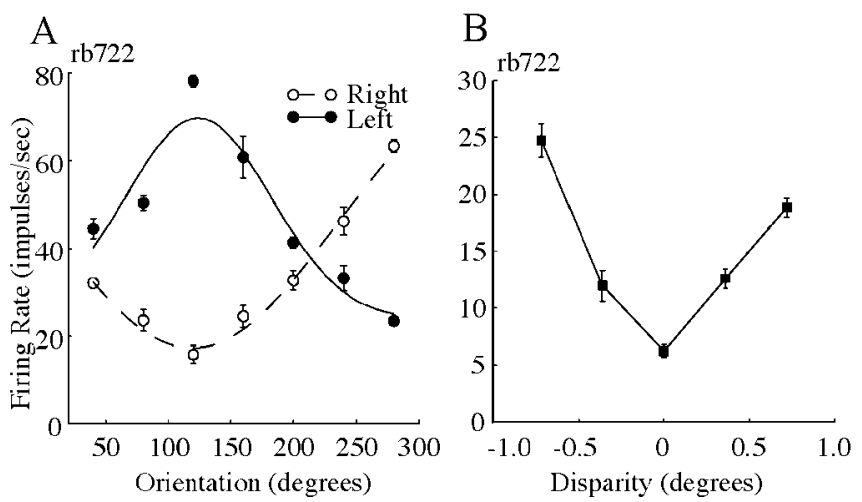

Figure 4. A, One of the three cases in which the left and right eyes showed opposite orientation tuning. The orientation that produced maximum excitation in one eye produced maximum suppression in the other eye. In these cases, Gaussian curves have been fitted to both eyes, but an inversion of the curve has been permitted to best describe the data. $B$, The disparity tuning curve measured using a sine-wave grating for this cell. The minimum response occurs at zero disparity because cell firing is suppressed when both eyes receive the same stimulus.

eye is inhibitory, whereas stimulation of the other eye is excitatory, but that the underlying orientation of the receptive field is in fact similar in the two eyes. For this reason, the fitted Gaussian was allowed to be inverted, and the cell shown in Figure 4 was deemed to have a small orientation difference.

Bandwidths were calculated by taking half width at half height of the fitted Gaussian curves. The average bandwidth for left and right eyes is $21.64 \pm 13.09^{\circ}$ and $19.92 \pm 10.84^{\circ}$, respectively. As shown in Figure 5, bandwidths in the left and right eyes are significantly correlated $(r=0.33 ; p<0.05)$, although not as highly 


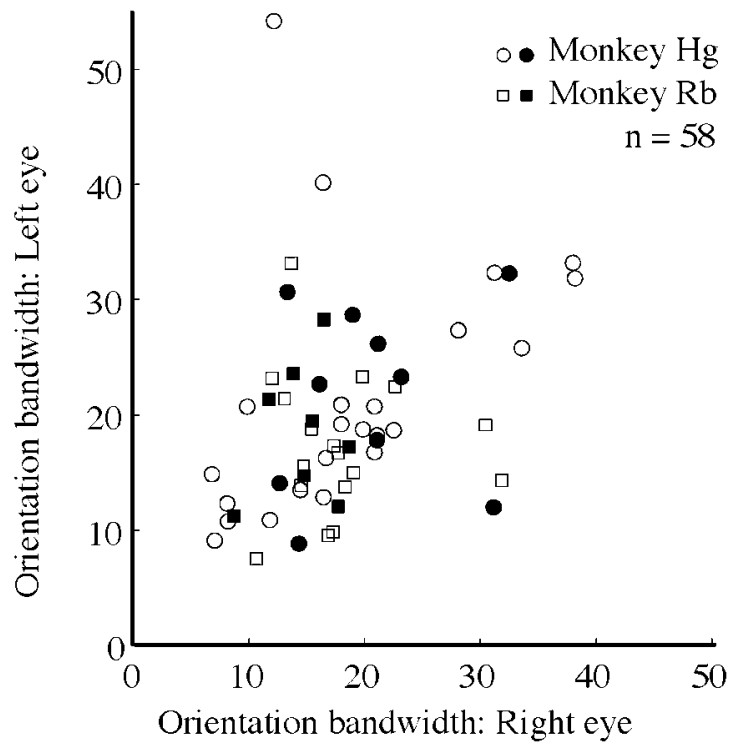

Figure 5. Left and right eye orientation bandwidths are significantly correlated $(r=0.33 ; p<0.05)$. Neurons with a significant IDPO are represented with filled circles, and those with no IDPO are shown as open circles. The average bandwidths for the left and right eyes are $21.64 \pm$ $13.09^{\circ}$ and $19.92 \pm 10.84^{\circ}$, respectively.

correlated as left and right preferred orientation. The three neurons that were fit with an inverted Gaussian in one eye were excluded from this analysis, because the meaning of bandwidth in this case is not clear.

The determination of complex or simple receptive field types in the awake monkey is complicated by small eye movements. There is no consensus as to how best to make this distinction, and no method has yet been subject to the close scrutiny that has been applied to work using anesthetized animals (Skottun et al., 1991). We used the method of Cumming et al. (1999); responses to drifting gratings were analyzed, but stimulus cycles during which a saccade was made were discarded. Cells in which the F1:F0 harmonic ratio that was estimated using this method was greater than one were classified as simple, following Skottun et al. (1991). However, cells with an F1:F0 harmonic ratio less than one could be either complex cells or simple cells in which the response has been confounded by a residual eye movement. Using this method, the mean bandwidth for the 15 simple cells was $17.94 \pm 6.98^{\circ}$ for the left eye and $19.04 \pm 7.18^{\circ}$ for the right eye. The corresponding values for the 43 complex cells were $21.33 \pm 9.17^{\circ}$ and $18.20 \pm$ $8.13^{\circ}$.

It is worth noting that there was no significant correlation between the measured magnitude of the IDPO and the orientation bandwidth $(r=0.14 ; p>0.05)$. This suggests that the IDPO measures do not simply reflect measurement error (which would produce larger differences when the bandwidth is larger).

\section{Binocular responses to orientation disparities}

The results in the preceding section confirm that interocular differences in receptive field (RF) orientation are present in monkey V1, as they are in the cat. This does not demonstrate that these IDPOs yield a useful binocular signal about orientation disparities. We examined this question with binocular stimuli containing orientation disparities. Binocular responses were examined in 81 of the 103 cells that showed significant monocular orientation tuning. The other 22 cells were lost after the monoc-
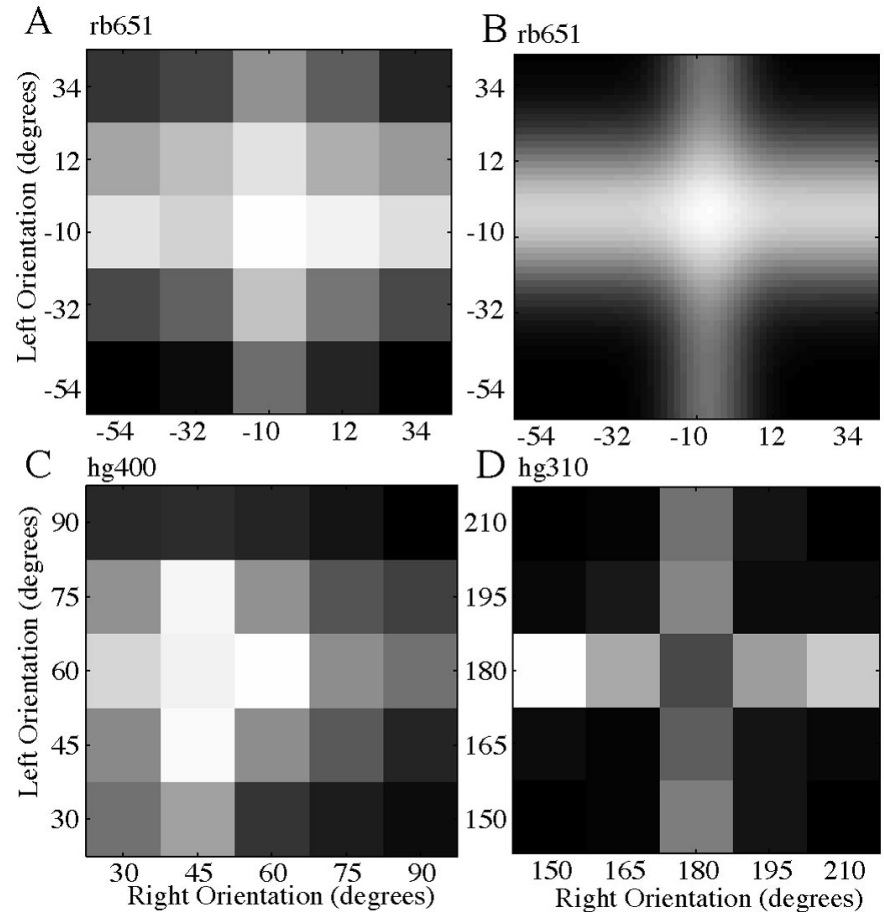

Figure 6. Three different types of left-right separable response patterns to binocular orientation differences. The level of brightness represents the rate of firing of the neuron; white areas represent high firing rates, whereas dark areas correspond to low firing rates. The most common type of response was that shown in $A$, with a central peak representing zero orientation disparity. $B$ shows the surface that is fitted to the data. The neuron has the same orientation preference for stimulation of either eye in $A$. In $C$, the monocular preferred orientations are different, and the maximum response occurs when the two eyes are receiving different orientations. The pattern shown in $D$ occurred in four neurons and is still left-right separable. The cell is activated when either eye receives its preferred orientation, but it is suppressed when both eyes receive the preferred orientation. Positional disparity tuning data were available in three of these cases, and they all exhibited tuned inhibitory disparity tuning.

ular orientation tuning curves were measured in each eye, but before the binocular experiment could be completed. The analysis of binocular orientation disparity encoding depends on fitting the data with one of Equations 4, 5, or 8 (see Materials and Methods). For 17 of 81 cells, all of these fits were poor $(<75 \%$ of variance explained by fit), so the analysis could not be applied to these cells. Many neurons for which the fits to binocular data were poor also showed irregular tuning to monocular stimuli; in 11 of the 17 cases, at least one of the monocular tuning curves was poorly fit by a Gaussian ( $<75 \%$ of variance explained by the fit). After exclusion of these 17 cells, there were 64 cells with adequate data that were well described by our equations.

The most common pattern of results is illustrated in Figure 6, $A$ and $C$. In these surface plots, the neuronal firing rate is represented by brightness; low firing rates are shown as dark patches, and the highest firing rate is shown as white. The responses are left-right separable. Although the response rate depends on the stimulus orientation in both eyes, the orientation that elicits the maximum response in one eye is independent of the stimulus orientation in the other eye. All of the neurons showing this pattern of results can be fit by a sum and product of Gaussians (Eq. 4). Adding the extra rotation term of Equation 5 did not produce a significant improvement $(p>0.05)$ in these 
Figure 7. This figure relates the surface plots to more conventional tuning curves. The monocular orientation tuning curves in $A$ predict that the maximum binocular response to orientation differences should occur when both eyes receive $90^{\circ}$. The data in $B$ is left-right separable because the preferred orientation in the right eye does not change when the orientation changes in the left eye. This is readily seen in the two cross sections through the surface shown in $C$. The cross section with the solid line shows the tuning to right eye orientation when the left eye orientation is kept constant at $90^{\circ}$. The maximum response

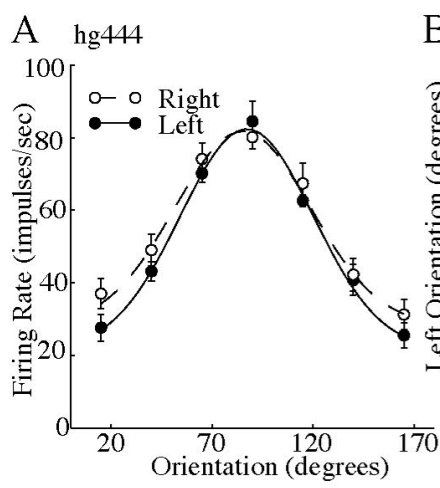

B hg444

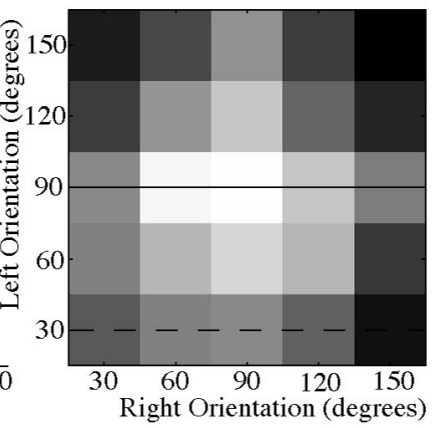

C hg444

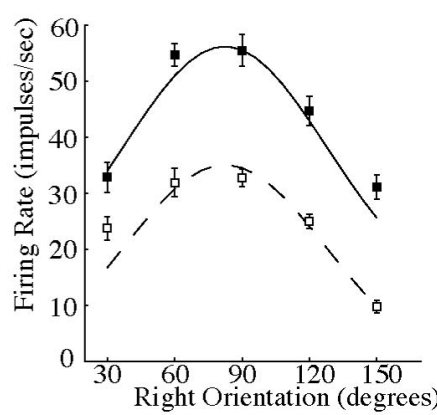
occurs when the right eye receives a

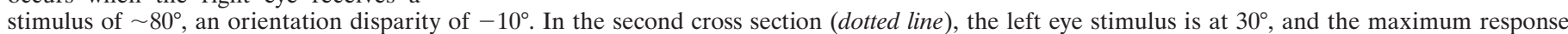

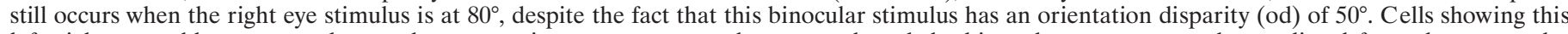

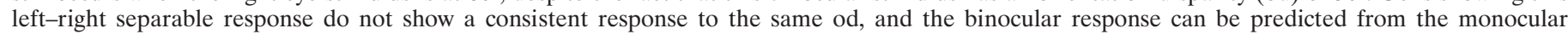
orientation tuning.

neurons. Figure $6 D$ shows an example in which there is an inhibitory binocular interaction, but the response is still left-right separable, so this is not specific to the orientation difference. Such a pattern was found in four cells. The three cells for which disparity tuning curves were available all showed a "tuned inhibitory" pattern of responses (Poggio and Fischer, 1977) to positional disparity (like that illustrated in Fig. $4 B$ ). Both of these patterns can be well fit by Equation 4. The fit to Figure $6 A$ is shown in $B$.

This common type of response that is left-right separable does not represent a consistent response to orientation disparities. This is highlighted in Figure $7 B$, which shows two cross sections through the two-dimensional surface plot in $A$ and the fit to this surface using Equation 4. It is obvious that the maximum response depends on the monocular orientation tuning, not the orientation disparity. The maximum response occurs when the right eye receives $80^{\circ}$ regardless of whether the left eye stimulus is at $90^{\circ}$, where it represents an orientation disparity of $-10^{\circ}$, or $30^{\circ}$, an orientation disparity of $+50^{\circ}$.

In addition to the cells described above, there is a further population that does appear to respond consistently to the same orientation disparity, regardless of the absolute orientation of the left and right stimuli; these responses were left-right inseparable. An example of such a cell is shown in Figure $8 A$. In this example, the maximum response occurs when both eyes receive the same orientation; it appears to be selective for an orientation disparity of zero. This pattern of response is left-right inseparable, and the fit to these data (Fig. $8 \mathrm{~B}$ ) is achieved by adding a rotation term to Equation 4 . The rotated Gaussian yields a significantly better fit ( $F$ test, $p<0.05)$ than the left-right separable Gaussian. The neuron response in Figure $8 C$ was also better fit $(C)$ with a rotated Gaussian, and the preferred IDPO is $\sim 30^{\circ}$. A total of 20 neurons showed left-right inseparable response profiles, which fell into two groups. One group (10 neurons) were fit with the inseparable Gaussian model (Eq. 5, the rotated form of the separable response profile). These tended to respond maximally for orientation disparities near zero, as illustrated in Figure $8 A$. The second group of 10 cells tended to show a consistent response minimum for orientation disparities of $0^{\circ}$, with two peaks either side, like the example in Figure $8 E$. Such response profiles cannot be well described by the rotated Gaussian of Equation 5 (this model accounted for $<75 \%$ of the response variance), and so Equation 8 was used, which provided a much better fit. This fit accounted
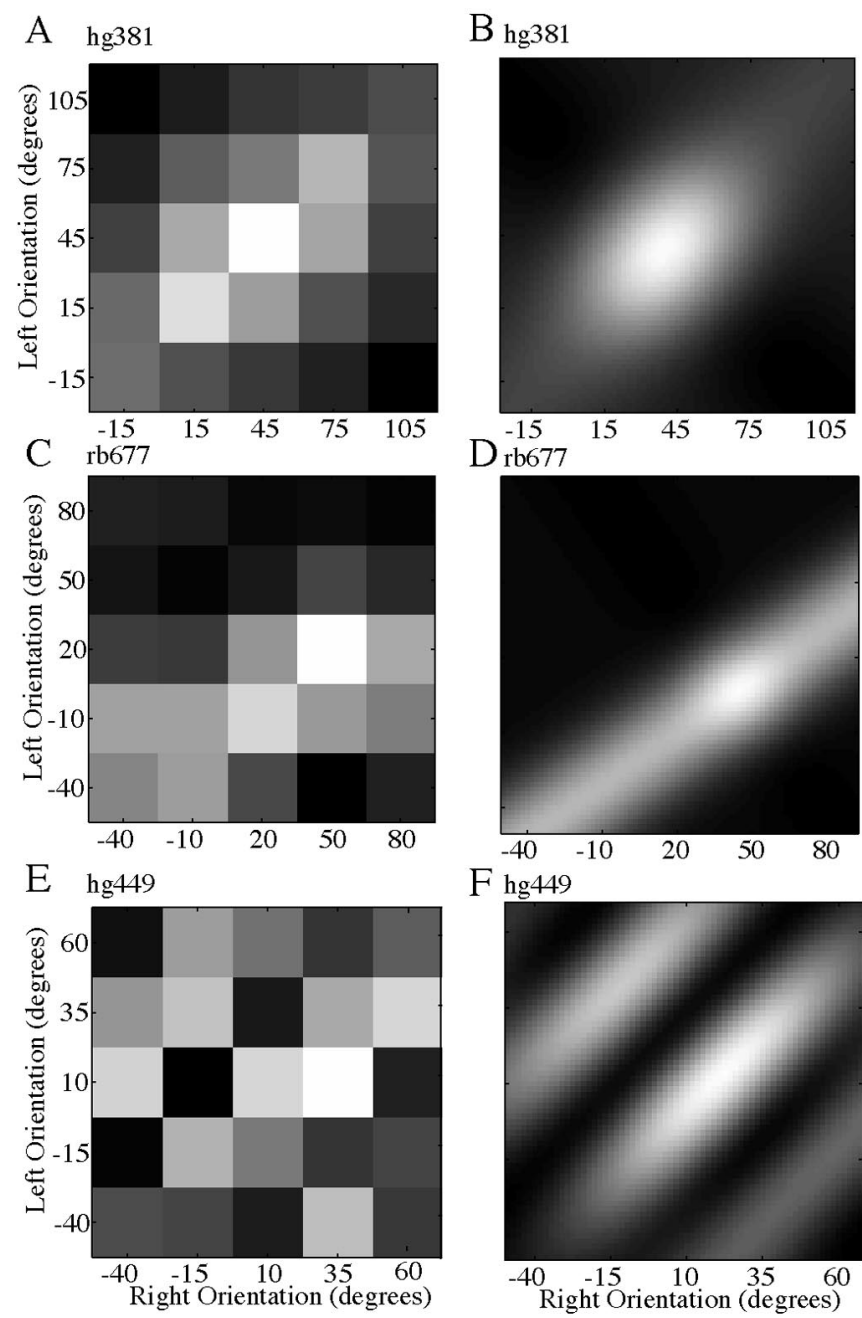

F hg449

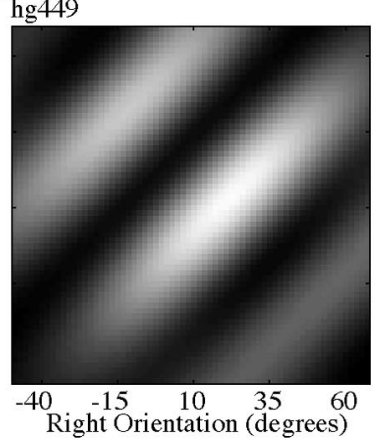

Figure 8. Three examples of cells that appear to respond consistently to orientation differences. In each pair, the left panel shows the raw data, and the right panel is the surface fitted to that data. The neuron in $A$ responds maximally when both eyes are receiving the same orientation. $C$ shows a neuron that consistently responded to a non-zero od. Both data sets were fitted using Equation 5. The multiple peaks and troughs in the data of $E$ meant that Equation 5 was not adequate to describe the data, and Equation 8 was required. 

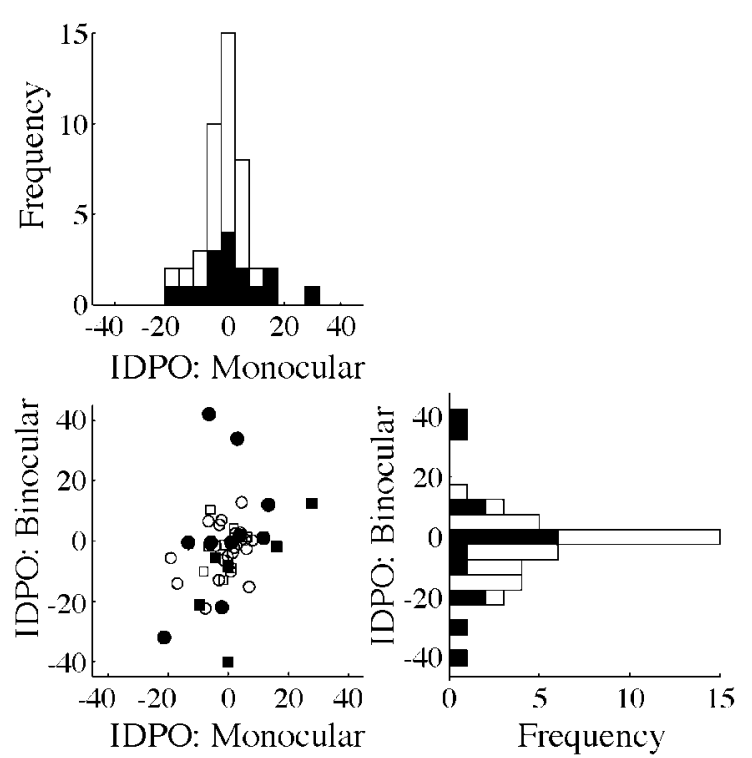

Figure 9. This figure illustrates the relationship between monocular and binocular measures of the IDPO for neurons that are significantly tuned to monocular orientation in both eyes. The histograms are the projection of the monocular and binocular differences. Cells that show separable and inseparable responses are represented as open and filled symbols, respectively. To enter this analysis, a good fit is required for both monocular orientation tuning curves and the binocular orientation disparity tuning, so the number of cells in the plot is reduced to 45. As in previous plots, neurons for monkeys $\mathrm{Hg}$ and $\mathrm{Rb}$ are shown with circles and squares, respectively.

for a substantially greater fraction of the response variance than the inseparable model or the separable model described by Equation 5.

To summarize, of the 64 neurons that could be well fit with one of the two surfaces, 44 showed the left-right separable response shown in Figure 6, and 20 were left-right inseparable. These left-right inseparable responses all indicate a tendency to respond consistently to a certain orientation disparity, regardless of the orientation of the stimulus in either eye alone. This suggests a specialization for signaling orientation disparities. However, several observations indicate that there may be an alternative explanation. If neurons are selective for orientation disparity, one would expect that the preferred orientation disparity measured with binocular stimuli would be similar to the difference in RF orientation determined from monocular measures. Figure 9 shows that this is not the case. The monocular measure was calculated from the difference in the peaks of the Gaussians fitted to monocular tuning curves. The binocular measure is simply the orientation disparity of the peak in the fitted response profile. These two measures were not significantly correlated $(r=0.26$; $p>0.05 ; n=45)$.

\section{The effect of tuning for positional disparity}

One factor that could produce discrepancies between monocular and binocular measures is disparity tuning. This may influence the shape of the binocular interaction, without affecting preferred orientation measured monocularly. Figure 10 therefore plots the magnitude of the discrepancy against the extent of disparity tuning. It is clear that the largest discrepancies occur in disparity selective neurons. To quantify this, we measured the variance of the absolute value of the discrepancy between monocular and binocular IDPO. This was calculated separately for the group of

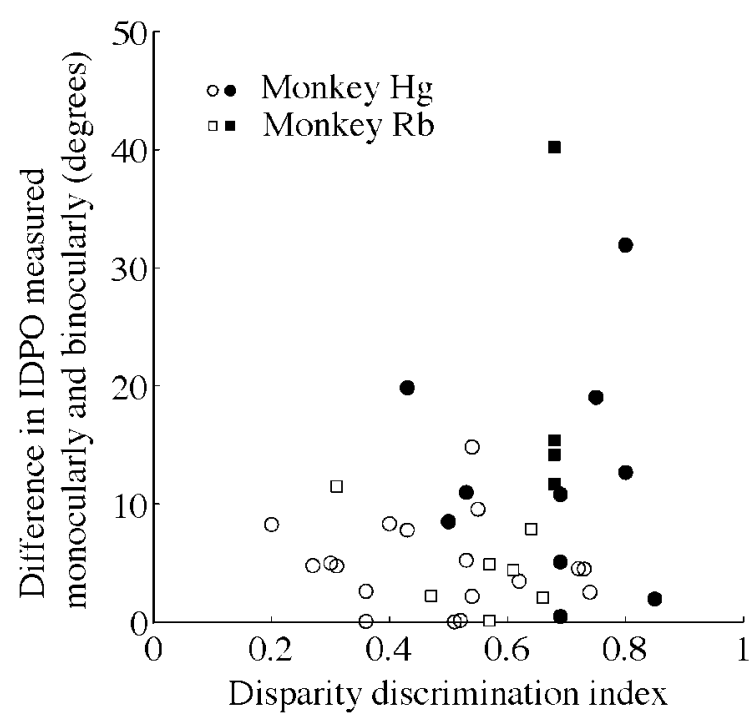

Figure 10. The relationship between the disparity discrimination index and the discrepancy between monocular and binocular measures of IDPO. Neurons that showed a left-right inseparable response to orientation disparities are shown with filled symbols; those showing a left-right separable response are shown with open symbols. The largest discrepancies are associated with stronger disparity tuning.

disparity selective neurons (determined by one-way ANOVA; $p<0.05)$ and for disparity unselective neurons. This variance was significantly larger for the disparity-selective neurons ( $F$ test; $p<$ $0.05)$.

This analysis is only possible in neurons that showed significant orientation tuning in both eyes. A similar analysis can be extended to the whole population by comparing monocular and binocular measures of the preferred stimulus orientation for each eye. The binocular measure is taken from the left and right orientations at the point of maximum binocular response, and the monocular measures are taken from the peaks of the Gaussian fit to the monocular tuning data. The relationship is examined in Figure 11, in which again it is clear that the largest discrepancies are associated with disparity selectivity. The correlation between the disparity discrimination index and the discrepancy in measures of preferred orientation is significant $(r=0.32 ; p<0.05)$.

Figure 11 also shows that the neurons exhibiting inseparable interactions between left and right stimulus orientations ( filled symbols) tend to show large discrepancies. The mean discrepancy between the monocular and binocular measures of preferred orientation is $4.8^{\circ}$ in neurons that showed separable interactions and $15.6^{\circ}$ in those that showed inseparable interactions.

It is also clear from Figures 10 and 11 that neurons exhibiting left-right inseparable responses tend to show disparity selectivity (significant at the 5\% level on a one-way ANOVA for 15 of 17 cases). In contrast, only 22 of the 40 cells that show a left-right separable response to binocular orientation disparities are disparity tuned. Together, Figures 9-11 strongly suggest that leftright inseparable responses like those illustrated in Figure 8 are in some way a result of tuning for positional disparity. In a few neurons, we explored this further by measuring binocular responses to orientation disparities with different positional disparities. Figure 12 shows one example in which two complete binocular interaction profiles were measured. Changing the stimulus disparity had a dramatic effect on the responses to orientation disparities, inverting the interaction profile. When the stimulus is 


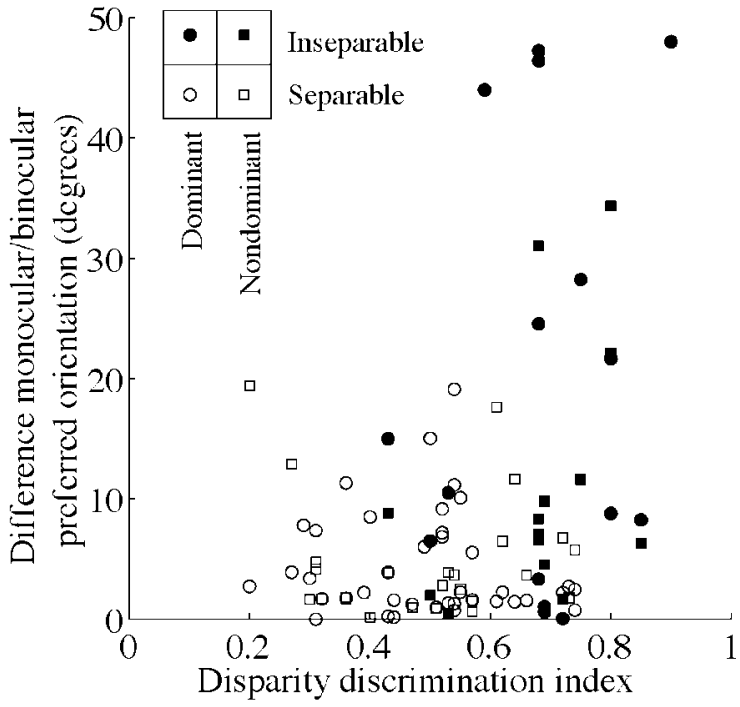

Figure 11. The discrepancy between monocular and binocular measures of preferred orientation, as a function of disparity selectivity. The preferred orientation is measured separately for the two eyes, so for all of those cells that are tuned for orientation in both eyes $(n=39)$, there are two points on the plot. The difference for the dominant eye is indicated by a circle, and the symbol for the difference of the nondominant eye is a square. In those cases where only one of the eyes is tuned for orientation $(n=16)$, a single point appears on the plot, also indicated by a circle. The discrepancy between the preferred orientation measured monocularly and binocularly is significantly correlated with the disparity discrimination index $(r=0.32 ; p<0.05)$.
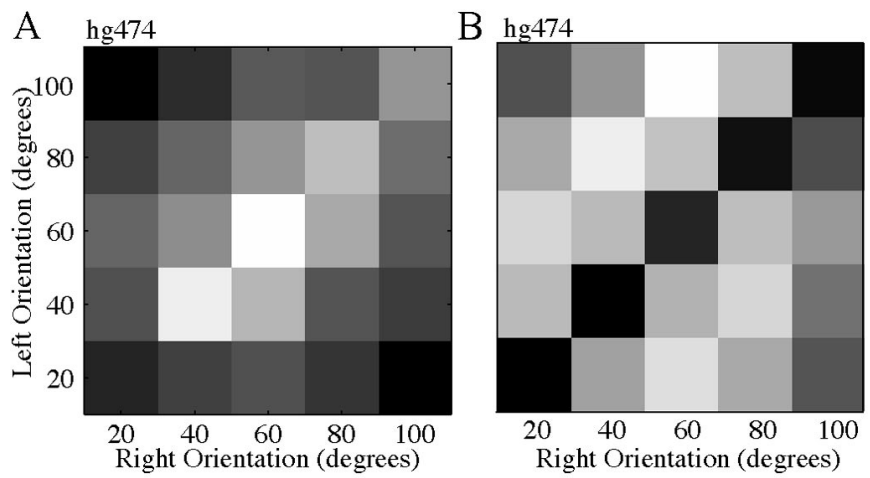

Figure 12. These are two binocular orientation disparity tuning curves recorded from the same cell. In $A$, the stimulus was at the preferred disparity of the cell $\left(-0.38^{\circ}\right)$, and in $B$, the stimulus was at a disparity to which the cell hardly responded $\left(0^{\circ}\right)$. If only $A$ were considered, it would appear that this cell responded consistently to $0^{\circ}$ orientation disparity. However, this excitation caused by $0^{\circ}$ orientation difference becomes inhibition when the stimulus disparity is changed in $B$.

at the preferred disparity of the neuron $\left(-0.38^{\circ}\right)$, the optimal orientation disparity is $0^{\circ}$. When the stimulus is at the positional disparity to which the neuron responds least $\left(0^{\circ}\right.$, the null disparity), the preferred orientation disparity is neither at $0^{\circ}$ nor is it predicted by the monocularly measured IDPO. Instead, stimuli with zero orientation disparity produce a minimum in the response.

The phenomenon illustrated in Figure 12 appears to be general in disparity-tuned neurons. In all cases in which there was an inseparable response that was selective for zero orientation disparity (seven neurons), the stimulus disparity turned out to be near to the preferred disparity of the neurons. In all cases in
A

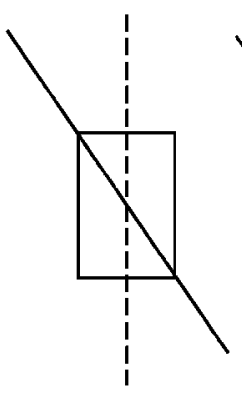

B

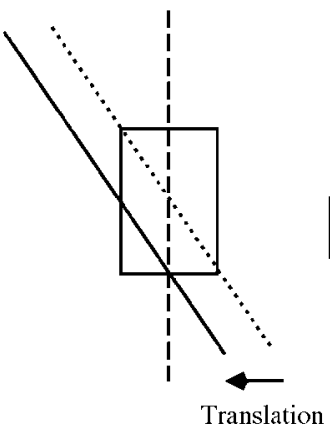

Right eye stimulus

Left eye stimulus

On-centre rotation

Receptive field

Figure 13. The schematic diagram in $A$ shows the stimulus on the receptive field when the rotation is performed about the center of the receptive field. The dashed line indicates the orientation of the left eye stimulus, and the solid line shows the orientation of the right eye stimulus. When the stimulus is rotated about the point at the bottom of the receptive field rather than the center $(B)$, there is a translation in the position of the right eye stimulus. Because this translation occurs only in the right eye, it is equivalent to a horizontal disparity. Arrow represents magnitude of horizontal disparity.

which there was a response minimum near $0^{\circ}$ orientation disparity, the disparity was near the null disparity of the neurons. We quantified this effect by calculating a simple index of how close a stimulus disparity $\left(d_{\text {stim }}\right)$ fell to the preferred disparity $\left(d_{\text {pref }}\right)$ as a proportion of the distance between preferred and null $\left(d_{\text {null }}\right)$

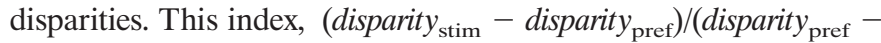
disparity $_{\text {null }}$, had a strong negative correlation with the magnitude of preferred orientation disparity determined from our fits $(r=-0.91$; $p \ll 0.01)$. This correlation between preferred positional disparity and preferred orientation disparity suggests that the positional disparity tuning determines the orientation disparity to which each neuron appears selective.

\section{The importance of where rotation is centered}

All of these observations (binocular IDPO is poorly correlated with monocular IDPO; the discrepancies are largest in disparitytuned neurons; and the preferred orientation disparity seems to depend on the positional disparity of the stimulus) suggest strongly that the left-right inseparable response comes about because of the positional disparity sensitivity of the neurons. One possible reason for this type of response is illustrated in Figure 13, which shows the consequences of applying an orientation disparity around a center of rotation that is not centered in the RF. The boxes represent vertically oriented receptive fields, the dotted line is the stimulus to the left eye, and the solid line is the stimulus to the right eye. For illustrative purposes, lines are used rather than sine-wave gratings, and the orientation disparity is produced by rotating the stimulus shown to the right eye only. On the left panel of Figure 13, the rotation occurs about the center of the receptive field. However, on the right, the rotation is about a point at the bottom of the receptive field. This is equivalent to the on-center rotation plus a translation. Because this translation is only applied to one eye, it constitutes a change in horizontal disparity. The magnitude of the horizontal disparity (illustrated by the arrow) will increase as the angle of stimulus rotation increases. Thus, off-center rotation leads to a complex interaction between binocular phase and binocular orientation differences.

To evaluate the effects of this interaction on our binocular measures of IDPO, we ran a simulation using the energy model of Ohzawa et al. (1990) (H. Bridge, B. G. Cumming, and A. J. 


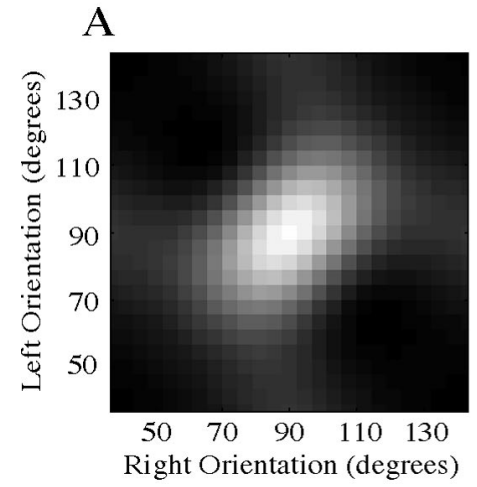

B

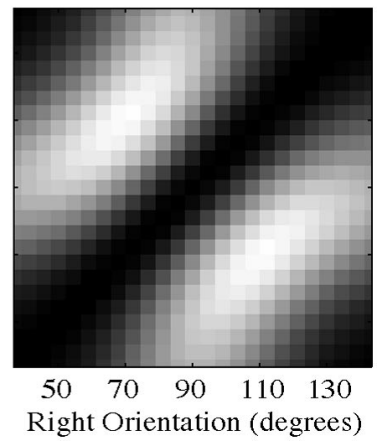

Figure 14. Rotating the stimulus about a point off the center of the receptive field causes a left-right inseparable response in the output of the energy model. When the stimulus is placed at the preferred positional disparity of the model cell $(A)$, there is excitation along the $0^{\circ}$ orientation difference diagonal. This line of excitation becomes inhibition when the disparity of the stimulus is changed to the null disparity of the model cell $(B)$.

Parker, unpublished observations). Our implementation extended the model to two-dimensional monocular receptive fields, so that the effects of both orientation differences and positional differences could be assessed. The results of this simulation are shown in Figure 14, in which two important features emerge. First, the combination of off-center rotation and disparity selectivity is sufficient to produce a response pattern that is left-right inseparable, in a way that closely resembles that of the single units. Second, the response pattern depends on the stimulus disparity. At the preferred disparity $(A)$, the maximum response occurs when the stimulus is at the preferred orientation in each eye. When the stimulus is at the null disparity, the same combination of orientations produces maximum inhibition $(B)$.

In summary, off-center rotation can, in principle, explain the left-right inseparable responses to binocularly measured IDPOs that we observed in V1 neurons. Furthermore, three specific characteristics of the data can be explained in the same way. First, maximum excitation at $0^{\circ}$ orientation disparity only occurs when the experiment has been performed with the stimulus at the preferred positional disparity, and conversely, there is a trough at $0^{\circ}$ orientation disparity when the stimulus is at the null disparity. Second, the discrepancy between the monocular and binocular measures of IDPO is greatest if the neurons are sensitive to positional disparity. Finally, the nature of the binocular tuning to orientation disparity changes with a change in the stimulus disparity as illustrated in Figures 12 (neuronal data) and 14 (energy model).

\section{DISCUSSION}

These experiments establish the presence of IDPOs in macaque V1 neurons using a statistical criterion. The use of an awake animal avoids the complication of torsional eye movements induced by anesthesia and paralysis. Nonetheless, we find a range of IDPOs similar to that reported previously from area 17 of the anesthetized cat (Blakemore et al., 1972; Nelson et al., 1977). Although doubts have been raised about the accuracy of the previous measures, this study provides unambiguous evidence of the presence and distribution of IDPOs.

The previous studies investigated only a limited range of binocular conditions. Here we examined responses to all combinations of left and right orientations, which enabled us to determine whether there was a specific binocular interaction that encoded the difference between the stimulus orientations in the two eyes.
Previous studies (Blakemore et al., 1972; Nelson et al., 1977; Wieniawa-Narkiewicz et al., 1992) kept the stimulus orientation constant in one eye. Those data are also compatible with a response pattern that was produced simply from summing left and right responses, with no mutual binocular interaction. Consequently, these earlier studies do not demonstrate a binocular mechanism for signaling orientation disparities or surface slant.

Around two-thirds (44 of 64) of the V1 neurons recorded showed a left-right separable response to binocular orientation disparities, whereas the other third (20 of 64) showed an inseparable response. However, several characteristics of this latter group suggested that their inseparable responses are in fact a consequence of positional disparity selectivity: (1) there is a poor correlation between the IDPO determined from monocular and binocular measures; (2) these discrepancies were largest in disparity-tuned neurons; (3) neurons showing inseparable responses were disparity-selective; (4) the preferred orientation disparity depended on the position disparity of the stimulus, in relation to the preferred disparity of the neuron; and (5) a simple model of disparity-selective neurons is able to reproduce all of these phenomena. The modeling suggests that the inseparable responses occur when the center of rotation for the orientation disparities is not located exactly at the center of the RF. For these reasons, we conclude that even the small number of neurons that showed left-right inseparable responses do not represent a mechanism specialized for signaling orientation disparities.

Another feature of the data that suggests that IDPOs are not important in signaling orientation disparities is the fact they are not correlated with orientation bandwidth. Because orientation disparities are usually small, cells with narrow orientation bandwidths are best suited to signaling naturally occurring orientation disparities. Our modeling work suggests that V1 neurons are best able to signal slant through detection of positional disparities. Only neurons with the narrowest orientation bandwidths could usefully exploit orientation disparities [although even these neurons signal slant more reliably via positional disparity (H. Bridge, B. G. Cumming, and A. J. Parker, unpublished observations)]. It is very unlikely that neurons with IDPOs and wide orientation bandwidths contribute to the detection of slant via orientation disparities. Because we find IDPOs are not limited to neurons with narrow orientation bandwidths, it is clear that the presence of an IDPO does not, by itself, suggest that a neuron is specialized for detecting orientation disparities.

These findings also represent a useful test of the energy model of disparity-selective complex cells (Ohzawa et al., 1990). In a modeling study, we show that the energy model predicts a leftright separable interaction between orientations, very similar to that illustrated in Figure $1 A$. In the energy model, an output nonlinearity renders neurons sensitive to the results of multiplying the left and right images. By combining the outputs of several simple cells with different phase sensitivity, this model generates responses to left and right eye locations that are left-right inseparable. Despite this, the interaction of different orientations is left-right separable, just as we observe here for real neurons. It is possible that V1 neurons could have encoded orientation and position separately, allowing them to respond to orientation disparities in ways quite different from those predicted by the energy model. Indeed, the idea that position and orientation are encoded separately at the monocular stage is implicit in the suggestion that orientation disparities represent a mechanism that is separate from position disparities. We found no evidence of this kind; all of the responses observed to both position and orientation dis- 
parities can be explained by supposing that a single monocular calculation is performed, which depends on both position and orientation. A simple convolution of the image with a fixed monocular RF, as in the energy model, is sufficient to account for the data. This lends further support to the view that the energy model provides a good description of the mechanism of disparity tuning in V1 (Cumming and DeAngelis, 2001).

\section{The role of cyclovergence}

Cyclovergence is the rotation of the two eyes in opposite directions about an axis from front to back through the center of the eye. If the stimuli used here elicited substantial cyclovergence movements, it would complicate the interpretation of these data. There are several reasons to suppose that such movements did not occur. Because the stimuli were matched in size and orientation to the RF, the orientation disparities applied did not produce a rotation centered on the fovea. Thus, these rotations are not a stimulus to cyclovergence. Even when the rotation is centered on the fovea, cyclovergence movements require large stimuli; Howard et al. (1994) found that decreasing stimulus size from 80 to $5^{\circ}$ led to a fivefold decrease in the gain of cyclovergence. Furthermore, they found that the central visual field in which our stimuli were placed made a small contribution to cyclovergence.

\section{The relationship between orientation differences and slant}

In considering the possible role of these neurons in signaling slant, it is important to recognize that on a real surface of fixed slant, the orientation difference is not uniform; it depends on the orientation of feature elements (Cagenello and Rogers, 1993). Vertical lines give rise to larger orientation differences than oblique lines. Nonetheless, a mechanism that signaled slant should still show left-right inseparability, with diagonal structure in a plot of responses to all left and right eye combinations. If this structure reflected real world geometry, the diagonal would not always be at $45^{\circ}$.

\section{Alternative ways of coding for slant}

Several recent studies in extrastriate cortex have reported neurons that appear to signal surface slant. Shikata et al. (1996) reported visual neurons in the parietal cortex that were selective for surface orientation in three dimensions. This same group later showed that cells in this area were selective not only for disparity gradient but also to texture gradient that corresponded to a similar surface orientation (Tsutsui et al., 1999). In addition to this data for the parietal cortex, Janssen et al. (1999) have described cells in the macaque inferior temporal cortex that are selective for the three-dimensional structure of surfaces. However, none of these studies addresses whether the binocular responses are the result of processing orientation disparities or the gradient of positional disparities. The neurophysiological data described here do not provide evidence for a mechanism that can signal orientation disparities. Rather, they suggest that the initial processing of disparity depends on the orientation and position of features in both eyes, in the way predicted by the energy model of Ohzawa et al. (1990). The proposal that there are two separate neural mechanisms early in visual processing (Blakemore et al., 1972), one that encodes orientation disparities and one that encodes position disparities, therefore has no physiological evidence to support it.

\section{REFERENCES}

Blakemore C, Fiorentini A, Maffei L (1972) A second neural mechanism of binocular depth discrimination. J Physiol (Lond) 226:725-749.

Cagenello R, Rogers B (1993) Anisotropies in the perception of stereoscopic surfaces: the role of orientation disparity. Vision Res 33:2189-2201.

Cumming B, Thomas O, Parker A, Hawken M (1999) Classification of simple and complex cells in (V1) of the awake monkey. Soc Neurosci Abstr 25:1548.

Cumming BG, DeAngelis GC (2001) The physiology of stereopsis. Annu Rev Neurosci 24:203-238.

Cumming BG, Parker AJ (1999) Binocular neurons in V1 of awake monkeys are selective for absolute, not relative, disparity. J Neurosci 19:1981-2088.

Cumming BG, Parker AJ (2000) Local disparity not perceived depth is signaled by binocular neurons in cortical area V1 of the macaque. J Neurosci 20:4758-4767.

DeValois RL, Yund EW, Hepler N (1982) The orientation and direction selectivity of cells in macaque visual cortex. Vision Res 22:531-544.

Draper NR, Smith HS (1998) Extra sums of squares and tests for several parameters being zero. In: Applied regression analysis, Ed 3, pp 149165. New York: Wiley.

Henry GH, Goodwin AW, Bishop PO (1978) Spatial summation of responses in receptive fields of simple cells in cat striate cortex. Exp Brain Res 32:245-266.

Howard I, Sun L, Shen X (1994) Cycloversion and cyclovergence: the effects of the area and position of the visual display. Exp Brain Res 100:509-514.

Hubel D, Wiesel T (1973) A re-examination of stereoscopic mechanisms in area 17 of the cat. J Physiol (Lond) 232:29P-30P

Janssen P, Vogels R, Orban G (1999) Macaque inferior temporal neurons are selective for disparity-defined three-dimensional shapes. Proc Natl Acad Sci USA 96:8217-8222.

Judge SJ, Richmond BJ, Chu FC (1980) Implantation of magnetic search coils for measurement of eye position: an improved method. Vision Res 30:535-538.

Merrill EG, Ainsworth A (1972) Glass-coated platinum-plated tungsten electrodes. Med Biol Eng 10:662-672.

Mitchison G, McKee S (1990) Mechanisms underlying the anisotropy of stereoscopic tilt perception. Vision Res 30:1781-1791.

Nelson J, Kato H, Bishop P (1977) Discrimination of orientation and position disparities by binocularly activated neurons in cat striate cortex. J Neurophysiol 40:260-283.

Ninio J (1985) Orientational versus horizontal disparity in the stereoscopic appreciation of slant. Perception 14:305-314.

Ohzawa I, DeAngelis GC, Freeman RD (1990) Stereoscopic depth discrimination in the visual cortex: neurons ideally suited as disparity detectors. Science 249:1037-1041.

Ohzawa I, DeAngelis GC, Freeman RD (1997) Encoding of binocular disparity by complex cells in the cat's visual cortex. J Neurophysiol 77:2879-2909.

Parker AJ, Hawken MJ (1988) Two-dimensional spatial structure of receptive fields in monkey striate cortex. J Opt Soc Am A 5:598-605.

Poggio GF, Fischer B (1977) Binocular interactions and depth sensitivity in striate and prestriate cortex of behaving rhesus monkey. J Neurophysiol 40:1392-1405.

Prince SJD, Pointon AD, Cumming BG, Parker AJ (2001) Quantitative analysis of responses of V1 neurons to horizontal disparity in dynamic random dot stereograms. J Neurophysiol, In press.

Shikata E, Tanaka Y, Nakamura H, Taira M, Sakata H (1996) Selectivity of the parietal visual neurones in 3D orientation of surface of stereoscopic stimuli. NeuroReport 7:2389-2394.

Skottun B, DeValois R, Grosof D, Movshon J, Albrecht D, Bonds A (1991) Classifying simple and complex cells on the basis of response modulation. Vision Res 31:1079-1086.

Tsutsui K, Taira M, Min J, Sakata H (1999) Coding of surface orientation by the gradient of texture and disparity in the monkey caudal intraparietal area. Soc Neurosci Abstr 25:670.

von der Heydt R (1978) Stereoscopic perception of orientation disparity. Invest Ophthalmol Vis Sci ARVO Abst 17:286.

von der Heydt R, Hanny P, Dursteler M (1980) The role of orientation disparity in stereoscopic perception and the development of binocular correspondence. Adv Physiol Sci 16:461-469.

Wheatstone C (1838) Contributions to the physiology of vision. I. On some remarkable, and hitherto unobserved, phenomena of vision. Philos Trans R Soc Lond B Biol Sci 13:371-395.

Wieniawa-Narkiewicz E, Wimborne B, Michalski A, Henry G (1992) Area 21a in the cat and the detection of binocular orientation disparity. Ophthalmic Physiol Opt 12:269-272. 